\title{
The Bacterial and Fungal Microbiota of Nelore Steers Is Dynamic Across the Gastrointestinal Tract and Its Fecal-Associated Microbiota Is Correlated to Feed Efficiency
}

\author{
Déborah Romaskevis Gomes Lopes ${ }^{1}$, Alex J. La Reau², Márcio de Souza Duarte³, \\ Edenio Detmann ${ }^{3}$, Cláudia Braga Pereira Bento', Maria Eugênia Zerlotti Mercadante ${ }^{4}$, \\ Sarah Figueiredo Martins Bonilha ${ }^{4}$, Garret Suen² and Hilario Cuquetto Mantovani ${ }^{\text {* }}$
}

\begin{abstract}
' Departamento de Microbiologia, Universidade Federal de Viçosa, Viçosa, Brazil, ${ }^{2}$ Department of Bacteriology, University of Wisconsin-Madison, Madison, WI, United States, ${ }^{3}$ Departamento de Zootecnia, Universidade Federal de Viçosa, Viçosa, Brazil, ${ }^{4}$ Instituto de Zootecnia, Centro APTA Bovinos de Corte, São Paulo, Brazil
\end{abstract}

OPEN ACCESS

Edited by:

Guillermina Hernandez-Raquet, Institut National de la Recherche

Agronomique (INRA), France

Reviewed by:

Stephan Schmitz-Esser, lowa State University, United States

Fuyong Li,

University of Alberta, Canada

Hui Yang,

Jiangxi Agricultural University, China

*Correspondence:

Hilario Cuquetto Mantovani hcm6@ufv.br

Specialty section: This article was submitted to Systems Microbiology, a section of the journal Frontiers in Microbiology

Received: 08 January 2019 Accepted: 21 May 2019

Published: 25 June 2019

Citation:

Lopes DRG, La Reau AJ, Duarte MS, Detmann E, Bento CBP, Mercadante MEZ, Bonilha SFM,

Suen G and Mantovani HC (2019) The Bacterial and Fungal Microbiota of Nelore Steers Is Dynamic Across the Gastrointestinal Tract and Its Fecal-Associated Microbiota Is

Correlated to Feed Efficiency. Front. Microbiol. 10:1263. doi: 10.3389/fmicb.2019.01263
The ruminant gastrointestinal tract (GIT) microbiome plays a major role in the health, physiology and production traits of the host. In this work, we characterized the bacterial and fungal microbiota of the rumen, small intestine (SI), cecum and feces of 27 Nelore steers using next-generation sequencing and evaluated biochemical parameters within the GIT segments. We found that only the bacterial microbiota clustered according to each GIT segment. Bacterial diversity and richness as well as volatile fatty acid concentration was lowest in the SI. Taxonomic grouping of bacterial operational taxonomic units (OTUs) revealed that Lachnospiraceae (24.61 \pm SD 6.58\%) and Ruminococcaceae (20.87 \pm SD 4.22\%) were the two most abundant taxa across the GIT. For the fungi, the family Neocallismastigaceae dominated in all GIT segments, with the genus Orpinomyces being the most abundant. Twenty-eight bacterial and six fungal OTUs were shared across all GIT segments in at least $50 \%$ of the steers. We also evaluated if the fecal-associated microbiota of steers showing negative and positive residual feed intake ( $n-R F I$ and $p-R F I$, respectively) was associated with their feed efficiency phenotype. Diversity indices for both bacterial and fungal fecal microbiota did not vary between the two feed efficiency groups. Differences in the fecal bacterial composition between high and low feed efficiency steers were primarily assigned to OTUs belonging to the families Lachnospiraceae and Ruminococcaceae and to the genus Prevotella. The fungal OTUs shared across the GIT did not vary between feed efficiency groups, but 7 and 3 OTUs were found only in steers with positive and negative RFI, respectively. These results provide further insights into the composition of the Nelore GIT microbiota, which could have implications for improving animal health and productivity. Our findings also reveal differences in fecal-associated bacterial OTUs between steers from different feed efficiency groups, suggesting that fecal sampling may represent a non-invasive strategy to link the bovine microbiota with productivity phenotypes.

Keywords: GIT microbiota, beef cattle, 16S rRNA gene, ITS1, fecal samples, residual feed intake, next-generation sequencing 


\section{INTRODUCTION}

Food-producing animals, such as monogastrics and ruminants, are responsible for most of the meat and milk produced worldwide, and these food products represent the main source of protein in the human diet. Brazil is one of the main producers of beef in the world, holding about $15 \%$ of the global meat exports (Food and Agriculture Organization of the United Nations [FAO], 2016). More than $90 \%$ of the Brazilian commercial cattle herds are animals belonging to the Nelore breed, a variety of Zebu (Bos indicus) that is well adapted to the environmental conditions and tropical production systems endemic to Brazil, thereby enabling production that is of global economic importance (Associação Brasileira das Indústrias Exportadoras de Carnes [ABIEC], 2017).

In order to make this production system more profitable and sustainable, farmers often seek strategies to decrease feeding costs through manipulation of rumen fermentation (e.g., ionophores) (Benatti et al., 2017; Crossland et al., 2017); altering management practices (e.g., supplements) (Jose Neto et al., 2016; Carvalho et al., 2017) and selection of cattle that have better feed conversion efficiencies (Nkrumah et al., 2006; Fidelis et al., 2017). Most of these practices target the ruminant gastrointestinal tract (GIT) microbiome and its function, as it is known to play a key role in the physiology of the mammalian host. For example, the GIT microbiota stimulates the immune system, produces vitamins and can inhibit pathogenic bacteria (Ley et al., 2006; Turnbaugh and Gordon, 2009; Cho and Blaser, 2012).

This association is even more relevant in ruminants since herbivorous animals are entirely dependent on symbiotic associations with anaerobic microorganisms from the GIT microbiota to digest plant cell wall polysaccharides (e.g., cellulose, hemicelluloses, and pectins) in the rumen (Krause and Russell, 1996). The rumen represents the main site for converting the ingested dietary components into energy to the host, while the microbiota colonizing the distal GIT (small and large intestine) are considered crucial for animal health, and to a lesser extent, the energy-harvesting functions of the host (Mao et al., 2015; Myer et al., 2015c).

Therefore, investigating the differences in microbial community composition across the ruminant GIT can provide further insights linking animal phenotypes and production traits with variation in microbial colonization and fermentation at different portions of the GIT. Previously, Oliveira et al. (2013) described the composition of the bacterial community in the GIT of a Nelore steer. However, this characterization was performed using a single animal, and changes in community composition were not linked to any specific phenotype. To gain a more in-depth understanding of the Nelore GIT microbiota, we investigated the bacterial and fungal composition of the rumen, small intestine (SI), cecum and feces of 27 Nelore steers showing differences in feed efficiency. Previous studies associating ruminant feed efficiency to microbial communities have focused primarily on the analysis of ruminal samples (McCann et al., 2014; Jewell et al., 2015; Shabat et al., 2016) whose collection depends on invasive methods or animal slaughter. Here, we investigated the compositional profile of the bacterial and fungal microbiota colonizing the Nelore GIT and also examined the hypothesis that steers with high and low feed efficiency show differences in their fecal microbiota.

Our results revealed that, despite variation in the composition of bacterial and fungal communities across different portions of the GIT, some OTUs known to be functionally relevant for fiber degradation and host development were shared across the entire GIT and present within the feces. Additionally, OTUs found in fecal samples were related to feed efficiency, suggesting that fecal microbiota analysis could be a practical method to assess differences in animal phenotypes, health status, or to monitor digestive disorders and the effect of diet, additives or supplements in cattle herds in a non-invasive manner.

\section{MATERIALS AND METHODS}

\section{Animals, Diets, and Sampling}

The experimental procedures were approved by the Ethics Committee on Animal Use of the Instituto de Zootecnia (CEUAIZ, Protocol 213-15), in accordance with guidelines of São Paulo State Law No. 11.977, Brazil, and by the Ethics Committee on Production Animal Use of the Universidade Federal de Viçosa (CEUAP-UFV, Protocol 026/2015).

Twenty-seven Nelore steers from the Centro Avançado de Pesquisa Tecnológica dos Agronegócios de bovinos de corte, a subsidiary of the Instituto de Zootecnia (São Paulo State, Brazil) and averaging $22.5 \pm$ SD 0.8 mo of age and $401 \pm$ SD $42 \mathrm{~kg}$ of BW were confined in individual pens $(4 \times 2 \mathrm{~m})$ with free access to the diet and water. These animals originated from a breeding program to select steers with improved individual performance and their residual feed intake (RFI) was identified during the growth period using the GrowSafe system, as previously described (Fidelis et al., 2017). For the finishing period, the steers were adapted to the diets, facilities, and management for 22 days and remained on the finishing diet for a 103-days period. The finishing diet was composed of $333 \mathrm{~g} / \mathrm{kg}$ corn silage, $17 \mathrm{~g} / \mathrm{kg}$ Brachiaria hay, $465 \mathrm{~g} / \mathrm{kg}$ dry ground corn, $163 \mathrm{~g} / \mathrm{kg}$ soybean meal, $6 \mathrm{~g} / \mathrm{kg}$ urea, $4 \mathrm{~g} / \mathrm{kg}$ ammonium sulfate, and $13 \mathrm{~g} / \mathrm{kg}$ mineral mixture (dry matter basis), formulated to meet the requirements of $1.3 \mathrm{~kg}$ of daily gain with a target finish weight of at least $550 \mathrm{~kg}$.

After finishing, steers were transported (130 km distance) to an experimental slaughterhouse (Pirassununga, São Paulo, Brazil). Fecal samples were collected the day before the slaughter and stored in sterile plastic containers at $-20^{\circ} \mathrm{C}$. Animal handling was conducted in accordance with good animal welfare practices, and slaughtering procedures followed strict guidelines established and regulated by the Sanitary and Industrial Inspection Regulation for Animal Origin Products, including a fasting period of $16 \mathrm{~h}$ (Brasil, 2017). After slaughter, the ruminal, intestinal (mid-jejunum) and cecal contents were collected in plastic sterile containers and stored at $-20^{\circ} \mathrm{C}$ for further analyses. Rumen samples were filtered through four layers of cheese cloth to separate the liquid from the solid fraction. 


\section{Concentration of Volatile Fatty Acids}

Organic acids were determined by HPLC using a Dionex Ultimate 3000 Dual detector HPLC (Dionex Corporation, Sunnyvale, CA, United States) coupled to a refractive index (RI) Shodex RI-101 maintained at $40^{\circ} \mathrm{C}$ using an ion exclusion column Phenomenex Rezex ROA, $300 \times 7.8 \mathrm{~mm}$ maintained at $45^{\circ} \mathrm{C}$. The mobile phase was prepared with $5 \mathrm{mmol} / \mathrm{l}$ sulfuric acid $\left(\mathrm{H}_{2} \mathrm{SO}_{4}\right)$ in ultrapure water, filtered $(0.22 \mu \mathrm{m})$ and degassed using a vacuum pump. The flow rate of the mobile phase was $0.7 \mathrm{ml} / \mathrm{min}$. The samples $(2.0 \mathrm{ml})$ were centrifuged $(12,000 \times \mathrm{g}$, $10 \mathrm{~min}$ ) and the cell-free supernatants were treated with $600 \mu \mathrm{l}$ of calcium hydroxide and $300 \mu \mathrm{l}$ of cupric sulfate as described by Siegfried et al. (1984). Samples were freeze/thawn and centrifuged $(12,000 \times g, 10 \mathrm{~min})$ three times before concentrated sulfuric acid $(28 \mu \mathrm{l})$ was added to the cell-free supernatants. Stock solutions of standards were prepared using the following organic acids: acetic, succinic, propionic, valeric, isovaleric, isobutyric, and butyric acid. All organic acids were prepared with a final concentration of $10 \mathrm{mmol} / \mathrm{l}$, except isovaleric acid (5 mmol/l) and acetic acid $(20 \mathrm{mmol} / \mathrm{l})$. Stock solutions were diluted 2-, 4-, 8-, and 16-fold in $5 \mathrm{mmol} / \mathrm{l} \mathrm{H}_{2} \mathrm{SO}_{4}$ and used as standards in the HPLC analysis.

\section{DNA Extraction and Sequencing}

Total genomic DNA from each sample was extracted following a mechanical disruption and phenol/chloroform extraction protocol described by Stevenson and Weimer (2007), which has been shown to generate high-quality, high-abundance DNA representative of complex bacterial community (Henderson et al., 2013). Extracted genomic DNA was quantified using a Nanodrop spectrophotometer (Thermo Scientific, Wilmington, DE, United States) and DNA was shipped for sequencing to the University of Wisconsin-Madison (United States).

The V4 hypervariable region of the bacterial 16S rRNA gene (length, ca. $250 \mathrm{bp}$ ) and the fungal internal transcribed spacer (ITS1; length, ca. $250 \mathrm{bp}$ ) were amplified using primers described by Kozich et al. (2013) and Kittelmann et al. (2013), respectively. Bacterial PCR reactions consisted of $50 \mathrm{ng}$ of the total DNA, $0.4 \mu \mathrm{M}$ of each primer, 1X Kapa Hifi HotStart ReadyMix (KAPA Biosystems), and water to $25 \mu \mathrm{l}$. For fungi, total DNA was increased to $100 \mathrm{ng}$ and primers to $1.6 \mu \mathrm{M}$ each. PCR was performed at $95^{\circ} \mathrm{C}$ for $3 \mathrm{~min}$, $95^{\circ} \mathrm{C}$ for $30 \mathrm{~s}, 55^{\circ} \mathrm{C}$ for $30 \mathrm{~s}, 72^{\circ} \mathrm{C}$ for $30 \mathrm{~s}(25$ cycles for $\mathrm{V} 4$ region and 35 cycles for ITS1) and a final extension step at $72^{\circ} \mathrm{C}$ for 5 min. PCR products were purified by PureLink $^{\circledR}$ Pro 96 PCR Purification Kit (Invitrogen, Carlsberg, CA, United States) and a second PCR was performed on the resulting amplicons to attach Illumina sequencing adapters and unique dual indices. PCR reactions were similar to those for V4 except that $5 \mu$ l of non-quantified PCR products were used as template DNA and 8 cycles were performed. PCR products were recovered by gel extraction in AquaPōr LM lowmelt agarose (National Diagnostics, Atlanta, GA, United States) using the Zymoclean Gel DNA Recovery Kit (Zymo Research, Irvine, CA, United States). Purified DNA was quantified by Qubit ${ }^{\circledR}$ Fluorometer (Invitrogen, Carlsbad, CA, United States) and equimolar amounts were pooled to create a single sample at $1 \times 10^{9} \mathrm{ng}$ per $\mu \mathrm{l}$ (Dias et al., 2017). Paired-end sequencing was performed using the v2 kit $(2 \times 250 \mathrm{bp})$ for V4 region and v3 kit $(2 \times 300 \mathrm{bp})$ for ITS1 on an Illumina MiSeq following manufacturer's guidelines (Illumina, Inc., San Diego, CA, United States). All DNA sequences have been deposited in the NCBI's Sequence Read Archive (SRA) under BioProject accession number PRJNA512996.

\section{Sequence Analysis}

Prior to analysis, all raw sequences obtained from the Illumina MiSeq were demultiplexed using the Illumina software system in order to remove sequencing adapters and low-quality base pair calls. Bacterial and fungal sequences were then processed separately using mothur (v1.39.5) following the MiSeq SOP (Schloss et al., 2009). Briefly, paired-end reads were joined using the make.contigs command with default parameters, and sequences shorter than 200 bp or longer than 500 bp for Bacteria and shorter than 200 bp or longer than $600 \mathrm{bp}$ for Fungi were removed. For both, sequences containing ambiguous characters or exhibiting a homopolymer run greater than 8 bp were removed. The V4 sequences were aligned using the SILVA 16S rRNA gene reference database release 128 (Quast et al., 2012) and the ITS1 sequences were aligned using the UNITE database (Kõljalg et al., 2013). Sequences that did not align to the correct location were removed. Identical sequences were grouped using the unique.seqs command and sequences that had two or fewer base pairs different were considered the same and grouped using pre.cluster. Chimeric sequences were detected using the UCHIME algorithm (Edgar et al., 2011) and removed. Singletons (sequences that occurred only once in the entire dataset) were also removed. Bacterial and fungal sequences were taxonomically assigned using classify.seqs to the SILVA and UNITE gene reference databases, respectively, with a bootstrap cut-off of 80 . The method of Naive Bayesian Classifier (NBC) was used to find the taxonomy of query sequences (Wang et al., 2007). Bacterial and fungal sequences were grouped into operational taxonomic units (OTUs) using uncorrected pairwise distances clustered with the furthest neighbor method and the average neighbor method, respectively, with a similarity cut-off of $97 \%$. Before proceeding with analysis, the coverage of all samples was assessed by Good's coverage. Due to different sequencing depths, OTU tables were normalized to equal sequence counts $(5,122$ sequences for Bacteria and 1,707 for Fungi, established by the sample that presented the lowest number of sequences). The normalized OTU tables were used to determine alpha diversity indices (Chao1, Shannon and Simpson) and to calculate relative abundances of OTUs.

\section{Statistical Analysis}

Differences in bacterial and fungal alpha-diversity, as well as in VFA concentration across the GIT were performed in MiniTab $^{\circledR}$ 17.1.0 (Minitab, Inc., Quality Plaza, 1829 Pine Hall Road, State College, Pennsylvania 16801, United States) by ANOVA, followed by post hoc Tukey test. $P$-values below 0.05 were considered significant. To evaluate the clustering 
of steers using OTU composition across the GIT, a nonmetric multidimensional scaling (nMDS) analysis using the Bray-Curtis dissimilarity index (beta diversity index), and non-parametric analyses of similarities (ANOSIM, number of permutation $=10,000)$ were performed using the Past software package (Hammer et al., 2001).

Venn diagrams were constructed using the tools available in the "Bioinformatics and Evolutionary Genomics" website to visualize shared and exclusive OTUs ${ }^{1}$. These analyses were performed using OTUs that were present in at least 50\% (up to $100 \%$, according to the OTU being analized) of all steers analyzed in this study ( $\geq 13$ steers). This criteria was based on previous observations indicating that species of ruminal bacteria that exhibit a heritable component show high presence $(\geq 50 \%)$ across animals (Sasson et al., 2017).

Non-metric multidimensional scaling analysis was used to represent the beta-diversity of bacterial and fungal communities of fecal samples from steers showing positive ( $p$-RFI, low feed efficiency) and negative residual feed intake (n-RFI, high feed efficiency). To compare bacterial and fungal composition of the fecal samples from steers exhibiting either p-RFI or $\mathrm{n}$-RFI, Venn diagrams were constructed using the OTUs that were present in at least $50 \%$ (up to 100\%) of the steers belonging to each feed efficiency group ( $\geq 7$ animals to the p-RFI group and $\geq 6$ animals to the $n$-RFI group). The KolmogorovSmirnov, Shapiro-Wilk and D'Algostino and Pearson tests were performed using GraphPad Prism v. 5.00 (GraphPad Software, San Diego, CA, United States) to determine if the relative abundance of bacterial and fungal OTUs followed a Gaussian distribution. Since most OTUs did not follow this distribution $(P>0.05)$, the differences in relative abundances according to feed efficiency group were assessed by White's non-parametric $t$-test using the software package STAMP v 2.1.3 (Parks et al., 2014). $P$-values below 0.05 were considered significant. The bacterial shared OTUs were plotted on a volcano plot to visualize the differences in relative abundances of OTUs present in the n-RFI steers versus the p-RFI steers. To accomplish this, the fold change (ratio between $n$-RFI and $\mathrm{p}$-RFI relative abundance of each OTU) was assessed along with the $p$-Values obtained using White's non-parametric $t$-test to visualize the data. Adjustments for multiple comparisons were not performed due to the exploratory nature of this study. In addition, differences in alpha-diversity of fecal samples according to each feed efficiency group were assessed by $t$-tests in MiniTab ${ }^{\circledR}$ 17.1.0 (Minitab, Inc., Quality Plaza, 1829 Pine Hall Road, State College, Pennsylvania 16801, United States). P-values below 0.05 were considered significant.

\section{RESULTS}

\section{Sequencing}

In total, we generated 10,000,278 raw bacterial sequences with an average length of $253 \mathrm{bp}$ across all samples. For fungi, we generated 7,226,285 raw sequences with an average length of

${ }^{1}$ http://bioinformatics.psb.ugent.be/webtools/Venn/
$241 \mathrm{bp}$ and across all samples. After trimming, quality filtering and removal of chimeras, 5,697,607 (mean 42,204 \pm SD 45,615 to samples) and 3,676,969 (mean 28,068 \pm SD 19,009 to samples) high-quality bacterial and fungal sequences were obtained, respectively. Good's coverage across samples was $>97 \%$ for bacterial sequencing and $>90 \%$ for fungal sequencing, indicating that our sequencing efforts sufficiently covered the diversity of bacterial and fungal communities in rumen liquids (RL) and solids (RS), SI, cecum and feces. The summary of sequence counts and OTUs that passed the steps of filtering, clean up and normalization are shown in Supplementary Table S1. Only reads and OTUs present in at least $50 \%$ of all the steers in a specific RFI group ( $\geq 7$ animals in p-RFI and $\geq 6$ animals in $n$-RFI) were considered for analysis of the feed efficiency phenotype (*cut-off in Supplementary Table S1).

\section{The Bacterial Microbiota Changes Across the Gastrointestinal Tract}

The intra-community diversity (alpha diversity) varied across different portions of the GIT (Tukey's test, $P<0.05$ ). Maximum values of Chao richness and Shannon diversity were observed in the RL and RS communities (1,296 \pm SD 207 and 5.21 \pm SD 0.26, respectively), while minimal values of Chao richness (641 $\pm \mathrm{SD}$ $189)$ and Shannon's diversity (3.78 \pm SD 0.73$)$ were found in the SI. The Simpson diversity index showed the inverse trend, with maximum values observed in the SI community $(0.071 \pm \mathrm{SD}$ 0.042 ) and minimal values in the RL, RS, and cecal communities $(0.022 \pm$ SD 0.01) (Figure 1A).

Beta diversity analysis showed that the Bray-Curtis dissimilarities of the bacterial communities differed according to GIT segment (ANOSIM, $P<0.001$ ). Main differences were observed comprising the rumen (RL and RS fractions), the SI and the large intestine (cecum and feces), and the bacterial communities in the RL/RS and cecum/feces samples also differed from each other (ANOSIM, $P<0.001$ ) (Figure 2A).

Taxonomic analysis of the GIT bacterial communities revealed 5,230 unique OTUs (mean $551 \pm$ SD 188 per sample after normalization) that were assigned to 24 phyla, 50 classes, 84 orders, 163 families, and 402 genera. The unclassified group represented 1.66 ( \pm SD 1.01\%), 2.48 ( \pm SD 1.26\%), 2.91 ( \pm SD1.34\%), 5.19 ( \pm SD 1.89\%), and 27.65 ( \pm SD 5.18\%) of the OTUs that could not be assigned to any phylum, class, order, family or genus, respectively. Firmicutes represented the predominant phylum in the bacterial community across the GIT of Nelore steers (relative abundance $66.68 \pm$ SD 9.15\%), followed by Bacteroidetes in RL, RS, cecum and feces samples (relative abundance of $20.50 \pm \mathrm{SD} 1.53 \%, 20.57 \pm \mathrm{SD} 1.59$, $11.34 \pm \mathrm{SD} 1.55$, and $12.17 \pm \mathrm{SD} 1.74$, respectively) and members of the phylum Actinobacteria in SI samples $(13.90 \pm \mathrm{SD}$ 2.05\%) (Figure 3A).

The most represented families across the GIT included the Lachnospiraceae (24.61 \pm SD 6.58\%) and Ruminococcaceae (20.87 \pm SD 4.22\%). Prevotelaceae was also predominant in ruminal bacterial communities (10.06 \pm SD 2.25\%), but its abundance decreased $(P<0.05)$ in other portions of the GIT $(3.15 \pm \mathrm{SD} 0.76 \%)$ (Figure 3B). 


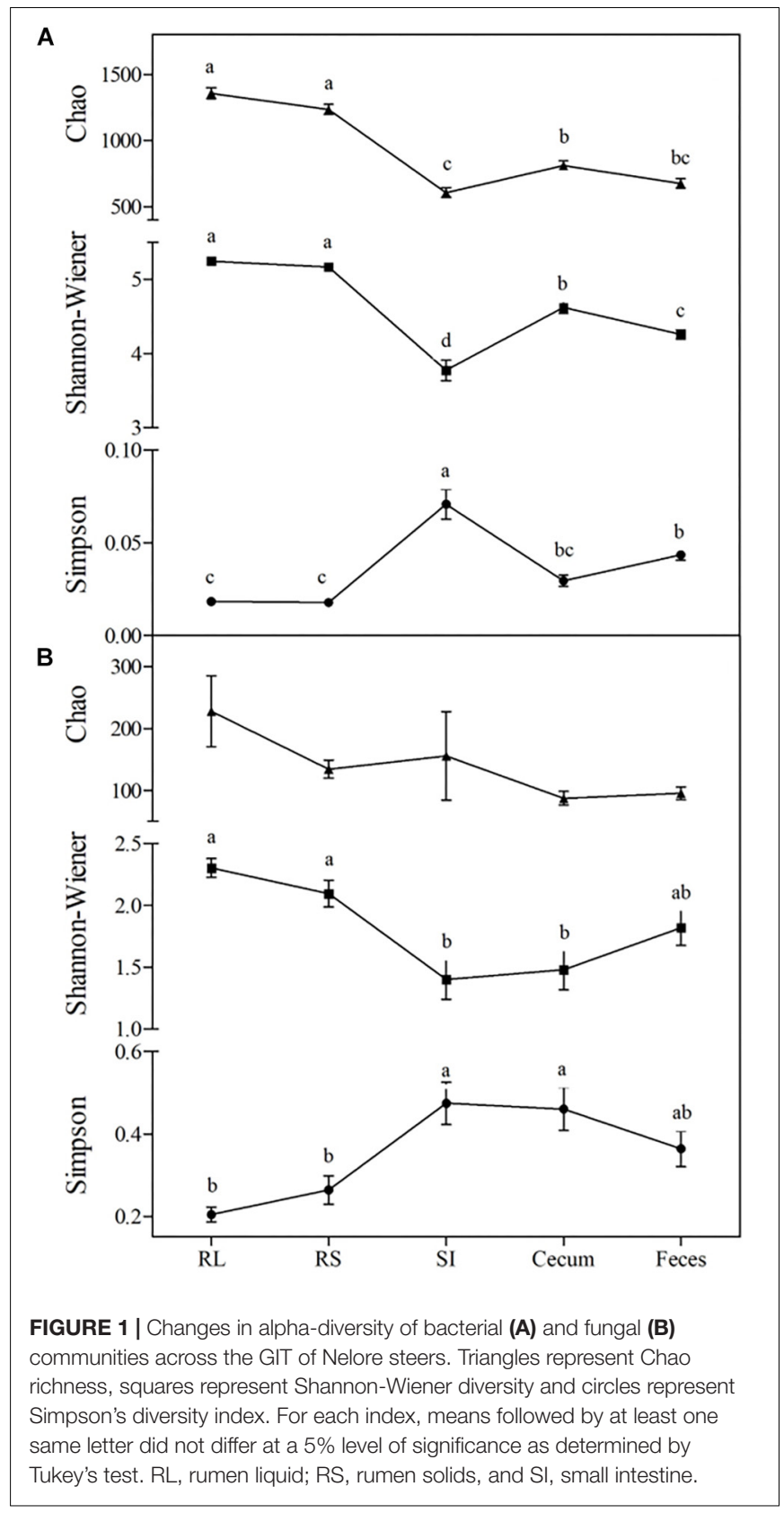

To filter the differences between the bacterial communities across the GIT, we used Venn diagrams to analyze OTUs from each portion of the GIT that were present in at least half of the Nelore steers ( $\geq 13$ steers for each portion of the GIT). We found 1,241 OTUs that were distributed across all samples, $9.91 \%$ which were exclusive to RL, $8.94 \%$ to RS, $10.56 \%$ to SI, $8.3 \%$ to cecum, and $4.03 \%$ to the feces (Figure 4A).

Taxonomic classification of the 28 OTUs shared across all 5 segments of the GIT showed that these sequences belonged to the Clostridiales (24 OTUs) and Coriobacteriales order (3 OTUs). One OTU shared by all GIT portions could not be assigned to any phylum. The highest classifiable level for the shared OTUs, as well the relative abundance across the GIT were represented in a heatmap (Figure 5). The majority of the shared OTUs showed higher abundances in the bacterial community colonizing the SI (with some OTUs also being more abundant in the RS community), but decreased in the large intestine with similar abundances in the cecum and feces. The most abundant OTUs in the SI were assigned to the order Clostridiales, with OTU00005 (genus Romboutsia, 10.51\%), OTU00007 (genus Paeniclostridium, 8.93\%), OTU00003 (genus Peptostreptococcaceae unclassified, 7.21\%), OTU00008 (genus Peptostreptococcaceae unclassified, 7.15\%), and OTU00009 (genus Lachnospiraceae NK3A20 group, 6.61\%) being the most representative ones.

\section{The Fungal Ruminal Microbiota Differs From the Distal Segment of the Gastrointestinal Tract}

The Shannon and Simpson indexes of the fungal community varied between the rumen and the distal (SI and cecum) segments of the GIT (Tukey's test, $P<0.05$ ). The maximum values of Shannon diversity and minimum values of Simpson's index were observed in the RL, RS, and fecal communities (2.07 $\pm \mathrm{SD} 0.61$ and $0.28 \pm \mathrm{SD} 0.18$, respectively). The fungal chao richness did not vary across the GIT (140 \pm SD 211, Tukey's test, $P>0.05$ ) (Figure 1B). The fungal beta diversity, summarized on a nMDS plot with Bray-Curtis dissimilarities, showed that the rumen fungal communities differed (ANOSIM, $P<0.001$ ) between the liquid and solid fractions (RS and $\mathrm{RL})$. In addition, the RS and RL fungal communities were also distinct from those in distal segments of the GIT (SI, cecum and fecal samples) (ANOSIM, $P<0.001$ ). However, it should be pointed out that no differences were observed between the fungal communities in the SI, cecum and fecal samples (ANOSIM, $P>0.001)$ (Figure 2B).

Taxonomic analysis of the GIT fungal communities revealed 3,253 unique OTUs (mean $62 \pm$ SD 34 per sample after normalization) that were assigned to 3 phyla, 6 classes, 6 orders, 8 families, and 12 genera. The unclassified group represented 13.47 ( \pm SD $10.28 \%), 13.77$ ( \pm SD $10.44 \%), 13.77$ ( \pm SD $10.44 \%), 15.65$ ( \pm SD $13.89 \%$ ) and 68.77 ( \pm SD $18.33 \%$ ) of the OTUs that could not be assigned to any phylum, class, order, family or genus, respectively. The OTUs assigned to the Neocallimastigaceae family represented more than $75 \%$ of the fungal OTUs across all segments of the GIT (Table 1).

Considering only the 31 fungal OTUs that were present in at least half of the Nelore steers ( $\geq 13$ steers), our Venn diagrams showed that $6.15 \%$ were exclusive to the RL, $3.23 \%$ to the RS, none to the SI, $3.23 \%$ to the cecum and $12.9 \%$ to the feces (Figure 4B). The six shared OTUs distributed between all five segments of the GIT of Nelore steers belonged to the Neocallimastigaceae (Figure 5).

\section{Microbial Fermentation Profile Changes Across the Gastrointestinal Tract}

Analysis of the microbial fermentation profiles across the GIT segments showed the highest concentration $(65.13 \mathrm{mmol} / \mathrm{l})$ of total volatile fatty acids (VFAs) in the cecum and feces, while 
A

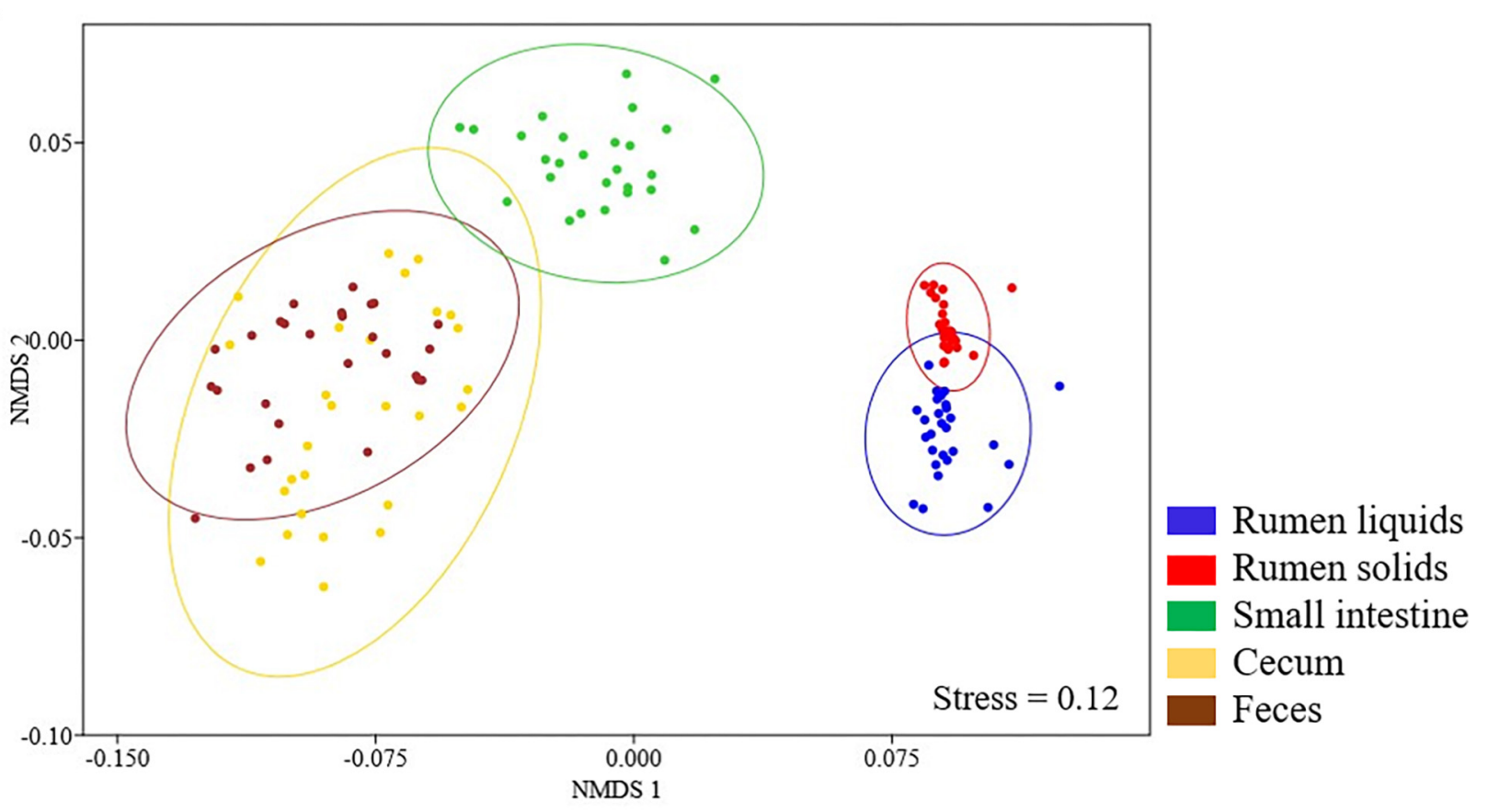

B

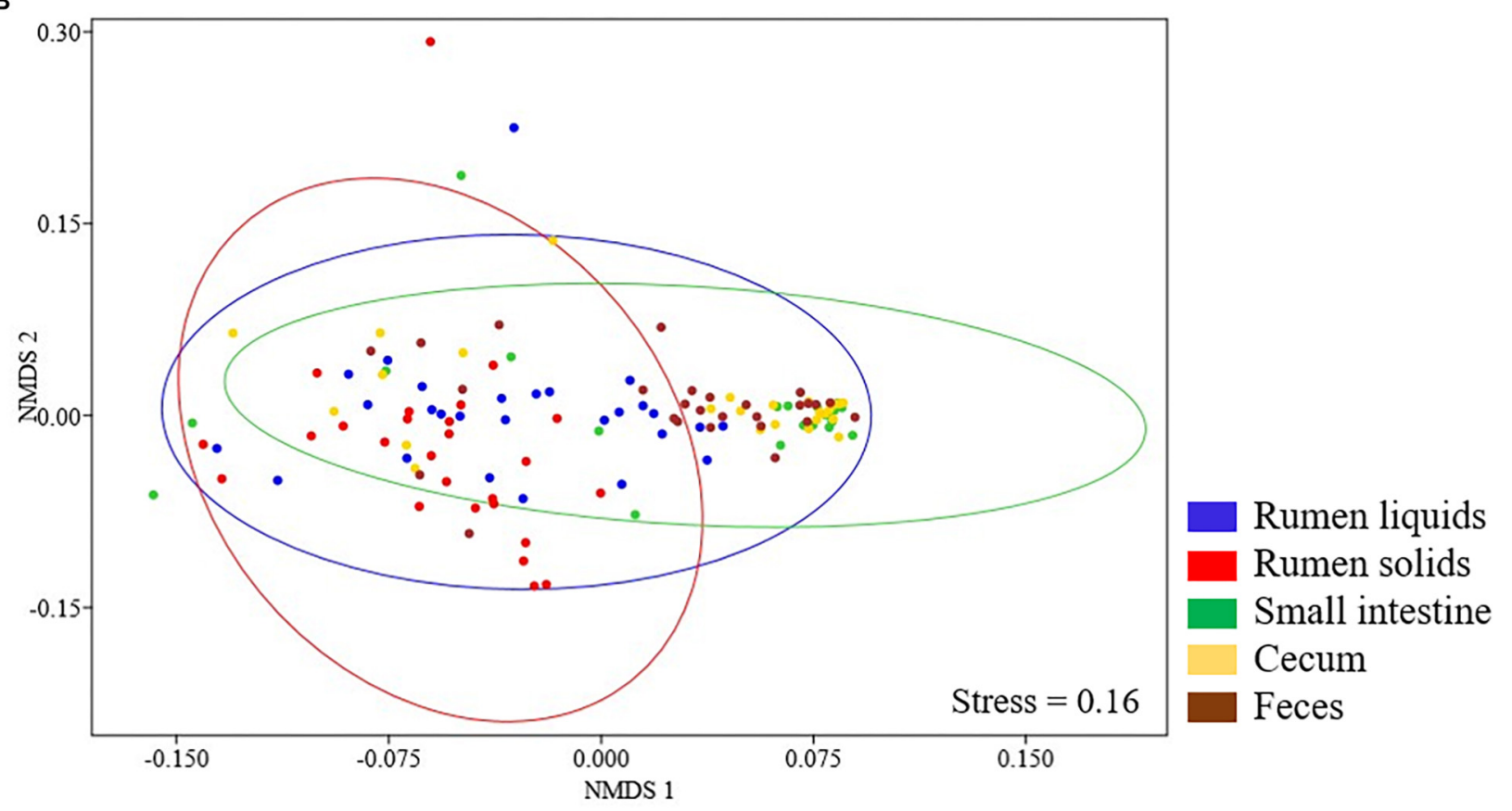

FIGURE 2 | Non-metric multidimensional scaling (nMDS) plots of the Bray-Curtis dissimilarity index for bacterial (A) and fungal (B) communities in the GIT of Nelore steers. Individual points represent GIT samples from different steers and different colors represent distinct GIT segments. Ellipses represent $95 \%$ confidence intervals.

the SI showed total VFA concentration almost six times lower $(11.57 \mathrm{mmol} / \mathrm{l})$ than other GIT segments (ANOVA, $P<0.05$ ) (Table 2). In the rumen, cecum and feces, the proportions of acetic, propionic and butyric acids were greater than other VFAs $(P<0.05)$. In the SI samples, succinic acid was present at a high proportion $(23.88 \%)$, in addition to acetic and propionic acids (Table 2).

\section{Specific Bacterial and Fungal OTUs in Fecal Samples Are Associated With the p-RFI and n-RFI Phenotype in Nelore Steers}

For the 27 steers used in this study, previous feed efficiency analysis performed during the growth period (approximately 

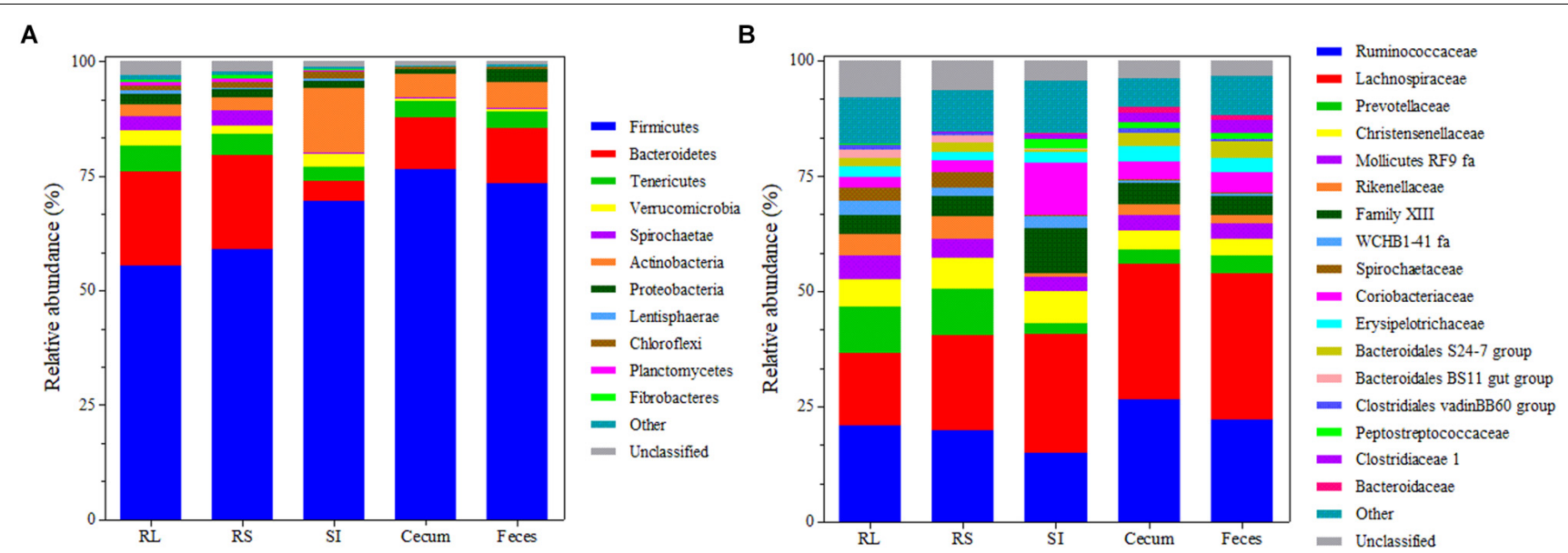

FIGURE 3 | Bacterial composition at the phylum (A) and family (B) level across the GIT of Nelore steers. Each bar represents the mean bacterial community composition in rumen liquids (RL), rumen solids (RS), small intestine (SI), and cecum and feces. For phylum, "other" corresponds to the sum of phyla that showed relative abundance $<0.5 \%$. For family, "other" corresponds to the sum of families that showed relative abundance $<1 \%$.
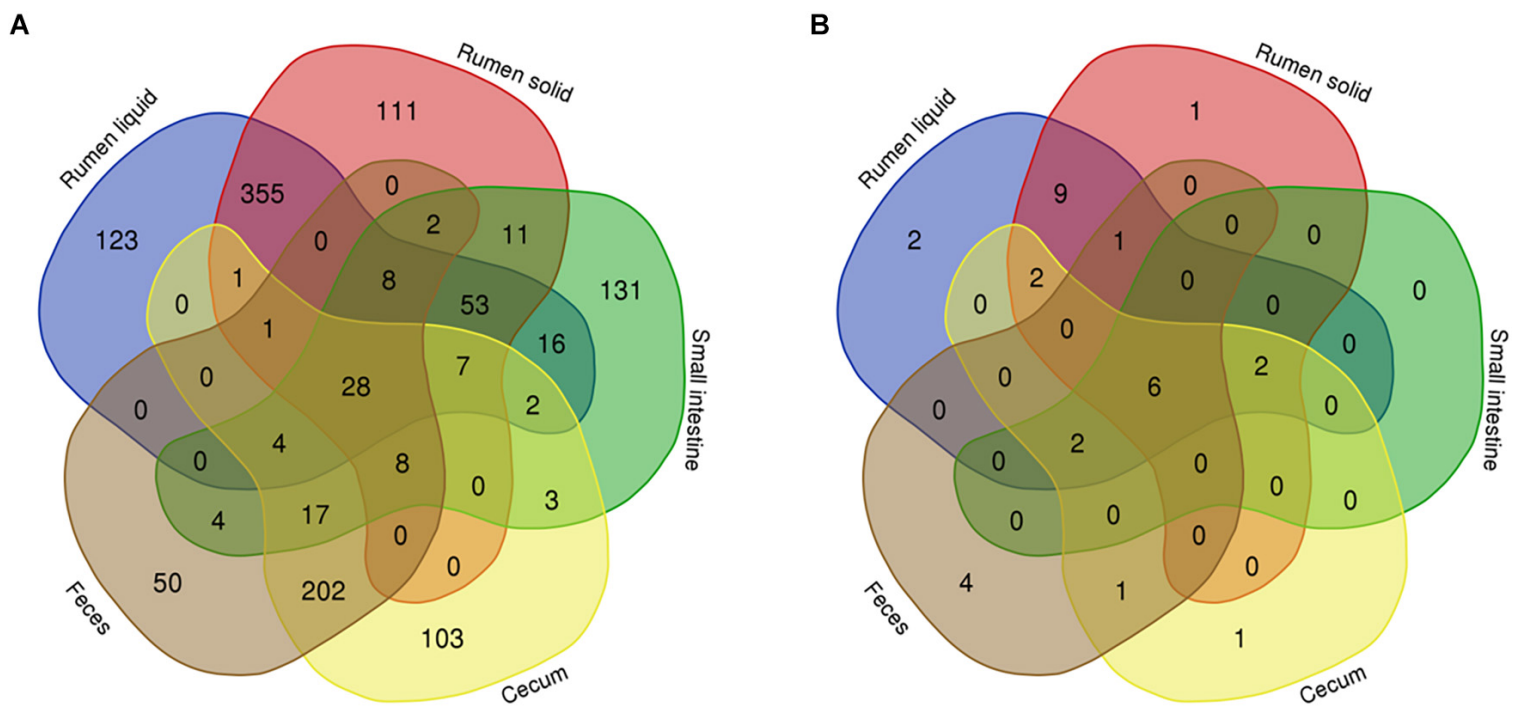

FIGURE 4 | Venn diagrams showing the number of bacterial (A) and fungal (B) OTUs shared between rumen (liquid and solids), small intestine, and cecum and feces. Only bacterial OTUs that were present in at least $50 \%$ of all steers (13 animals) from each GIT segment are represented.

12 months prior to sampling) identified 12 steers with n-RFI and 15 steers with positive RFI (p-RFI). The RFI values were significantly different $(P<0.05)$ between $n$-RFI $(-0.93 \pm \mathrm{SD}$ $0.17)$ and p-RFI steers $(0.87 \pm$ SD 0.14$)$, separating the animals into two groups: high and low feed efficiency, respectively. OTU analyses were conducted using fecal samples from these two feed efficiency groups to address the hypothesis that the fecal microbiome of n-RFI and p-RFI Nelore steers show differences in their bacterial and fungal community composition.

Alpha diversity analysis of the bacterial and fungal communities in the fecal samples showed that Chaol richness, Simpson's diversity, and Shannon's diversity did not vary significantly between RFI groups ( $t$-test $P>0.05$ ) (Supplementary Table S2). Analysis of beta diversity in fecal samples did not show differences (ANOSIM, $P>0.001$ ) between bacterial and fungal communities of $\mathrm{p}$-RFI and n-RFI steers (Supplementary Figure S1). However, a more detailed analysis of the OTUs present in at least half of the steers classified in each feed efficiency group ( $\geq 7$ animals to the p-RFI group and $\geq 6$ animals to the $n$-RFI group) using Venn diagrams showed bacterial and fungal OTUs that were unique to each efficiency group.

For the bacteria, 99 OTUs were unique to the p-RFI steers (corresponding to $5.16 \%$ of the total relative abundance), while the n-RFI steers had 41 unique OTUs representing 1.54\% of total relative abundance (Figure 6A). The most abundant bacterial OTUs present only in p-RFI or n-RFI steers were OTU0034 (Alloprevotella) and OTU00234 (Turicibacter), respectively 


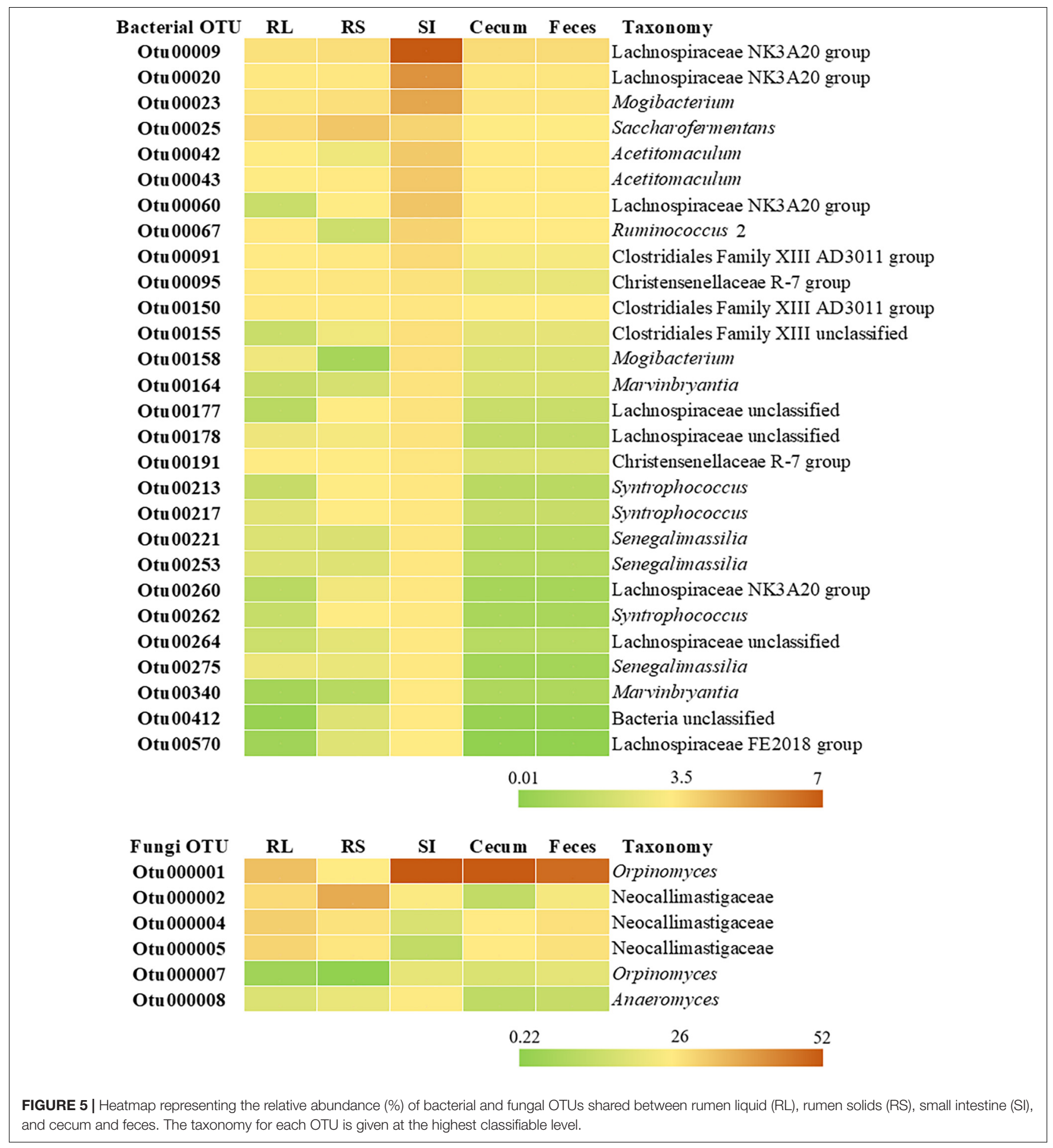

(Supplementary Table S3). Differences in relative abundance of the bacterial OTUs shared between the p-RFI and n-RFI groups (66.43\% of the total OTUs) were represented on a volcano plot (Figure 6B). Of the 277 shared OTUs, 46.57\% showed higher abundance in the n-RFI steers with only 3 OTUs significantly more abundant in these steers (White's non-parametric $t$-test, $P<0.05)$. For the p-RFI steers, $53.43 \%$ of the OTUs showed higher abundance in these steers, relative to the n-RFI group and only seven OTUs were found to be significantly more abundant (White's non-parametric $t$-test, $P<0.05$ ) (Figure 6B). Among the OTUs that were statistically more abundant in n-RFI steers, OTU00065 (unclassified Lachnospiraceae) represented almost $1 \%$ of the relative abundance of OTUs in this group. In the p-RFI steers, OTU00153 (Coprococcus) had relative abundances 
TABLE 1 | Fungal composition profile across the GIT of Nelore steers.

\begin{tabular}{|c|c|c|c|c|c|c|}
\hline Level & Taxa & Rumen liquids & Rumen solids & Small intestine & Cecum & Feces \\
\hline \multirow[t]{3}{*}{ Phylum } & Neocallimastigomycota & $85.51 \pm 11.88$ & $95.04 \pm 4.98$ & $86.71 \pm 13.23$ & $85.77 \pm 9.76$ & $77.29 \pm 12.35$ \\
\hline & Others & $0.32 \pm 0.36$ & $0.07 \pm 0.09$ & $0.47 \pm 1.91$ & $0.29 \pm 0.37$ & $1.16 \pm 1.69$ \\
\hline & Unclassified & $14.17 \pm 11.59$ & $4.89 \pm 4.91$ & $12.82 \pm 13.23$ & $13.94 \pm 9.56$ & $21.55 \pm 12.12$ \\
\hline \multirow[t]{3}{*}{ Class } & Neocallimastigomycetes & $85.51 \pm 11.88$ & $95.04 \pm 4.98$ & $86.71 \pm 13.72$ & $85.77 \pm 9.76$ & $77.29 \pm 12.35$ \\
\hline & Others & $0.00 \pm 0.00$ & $0.00 \pm 0.00$ & $0.39 \pm 1.83$ & $0.05 \pm 0.22$ & $0.41 \pm 1.76$ \\
\hline & Unclassified & $14.49 \pm 11.878$ & $4.96 \pm 4.98$ & $12.90 \pm 13.23$ & $14.18 \pm 9.75$ & $22.30 \pm 12.36$ \\
\hline \multirow[t]{3}{*}{ Order } & Neocallimastigales & $85.51 \pm 11.88$ & $95.04 \pm 4.98$ & $86.71 \pm 13.72$ & $85.77 \pm 9.76$ & $77.29 \pm 12.35$ \\
\hline & Others & $0.00 \pm 0.00$ & $0.00 \pm 0.00$ & $0.38 \pm 1.80$ & $0.05 \pm 0.22$ & $0.38 \pm 1.72$ \\
\hline & Unclassified & $14.49 \pm 11.88$ & $4.96 \pm 4.98$ & $12.91 \pm 13.23$ & $14.18 \pm 9.75$ & $22.33 \pm 12.36$ \\
\hline \multirow[t]{3}{*}{ Family } & Neocallimastigaceae & $83.22 \pm 15.35$ & $91.72 \pm 14.07$ & $84.88 \pm 15.13$ & $84.46 \pm 12.55$ & $76.61 \pm 12.39$ \\
\hline & Others & $0.00 \pm 0.00$ & $0.00 \pm 0.00$ & $0.38 \pm 1.80$ & $0.05 \pm 0.25$ & $0.38 \pm 1.75$ \\
\hline & Unclassified & $16.78 \pm 15.44$ & $8.28 \pm 14.10$ & $14.74 \pm 14.85$ & $15.49 \pm 12.61$ & $23.01 \pm 12.45$ \\
\hline \multirow[t]{6}{*}{ Genera } & Anaeromyces & $2.31 \pm 1.76$ & $1.65 \pm 0.63$ & $1.51 \pm 1.17$ & $0.98 \pm 0.81$ & $1.21 \pm 1.12$ \\
\hline & Cyllamyces & $0.34 \pm 1.11$ & $0.85 \pm 3.04$ & $1.52 \pm 6.46$ & $0.69 \pm 2.93$ & $0.29 \pm 1.30$ \\
\hline & Neocallimastix & $1.53 \pm 1.02$ & $1.42 \pm 0.74$ & $0.69 \pm 0.69$ & $0.42 \pm 0.39$ & $0.65 \pm 0.61$ \\
\hline & Orpinomyces & $13.38 \pm 12.58$ & $3.51 \pm 3.24$ & $44.15 \pm 29.85$ & $41.70 \pm 27.90$ & $36.18 \pm 22.09$ \\
\hline & Others & $0.11 \pm 0.19$ & $0.12 \pm 0.16$ & $0.45 \pm 1.94$ & $0.10 \pm 0.35$ & $0.40 \pm 1.80$ \\
\hline & Unclassified & $82.33 \pm 12.18$ & $92.45 \pm 4.73$ & $51.68 \pm 27.14$ & $56.11 \pm 26.48$ & $61.27 \pm 21.14$ \\
\hline
\end{tabular}

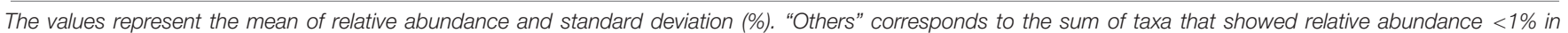
all TGl portions.

TABLE 2 | Fermentation profiles of Nelore steers GIT portions.

\begin{tabular}{|c|c|c|c|c|c|c|c|c|}
\hline \multirow[b]{2}{*}{ Parameter } & \multicolumn{2}{|c|}{ Rumen } & \multicolumn{2}{|c|}{ Small intestine } & \multicolumn{2}{|c|}{ Cecum } & \multicolumn{2}{|c|}{ Feces } \\
\hline & Mean & SEM & Mean & SEM & Mean & SEM & Mean & SEM \\
\hline Acetic acid(\%) & $69.36 a$ & 0.37 & $55.52 b$ & 2.99 & $65.95 a$ & 1.29 & $69.39 a$ & 0.85 \\
\hline Propionic acid(\%) & $14.51 \mathrm{a}$ & 0.38 & $16.71 \mathrm{a}$ & 2.41 & $15.65 a$ & 0.75 & $13.17 \mathrm{a}$ & 0.35 \\
\hline Butyric acid(\%) & $6.88 \mathrm{c}$ & 0.19 & $0.81 d$ & 0.29 & $9.01 b$ & 0.56 & $12.46 \mathrm{a}$ & 0.62 \\
\hline Isobutyric acid(\%) & $3.77 a b$ & 0.17 & $3.08 \mathrm{~b}$ & 0.70 & $5.82 a$ & 0.98 & $2.11 b$ & 0.18 \\
\hline Valeric acid(\%) & $0.97 b$ & 0.04 & - & - & $1.96 \mathrm{a}$ & 0.16 & $1.96 \mathrm{a}$ & 0.16 \\
\hline Isovaleric acid(\%) & $3.86 a$ & 0.19 & - & - & $1.60 \mathrm{~b}$ & 0.18 & $0.61 c$ & 0.07 \\
\hline Succinic acid(\%) & $0.65 b$ & 0.07 & $23.88 \mathrm{a}$ & 2.00 & - & - & $0.31 b$ & 0.11 \\
\hline A/P ratio* & $4.87 a b$ & 0.14 & $8.21 \mathrm{a}$ & 1.80 & $4.49 b$ & 0.24 & $5.40 a b$ & 0.19 \\
\hline Total VFa ** (mmol/l) & $51.80 b$ & 3.15 & $11.57 \mathrm{c}$ & 1.28 & $66.96 \mathrm{a}$ & 3.19 & $63.30 a$ & 3.25 \\
\hline
\end{tabular}

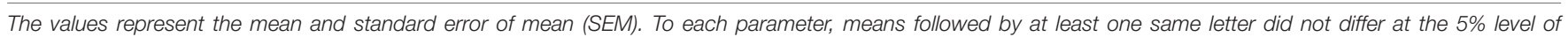
significance by the Tukey's test. *A/P, Acetic to propionic acid ratio; **, Total concentration of volatile fatty acids (VFAs); (-), not detected.

2.5 times greater in p-RFI than in n-RFI steers (Supplementary Table S4). For fungi, seven OTUs were unique to the fecal microbiome of $\mathrm{p}$-RFI steers (corresponding to $4.55 \%$ of relative abundance), while the n-RFI steers had three unique OTUS representing $1.14 \%$ of total relative abundance (Figure 7 and Supplementary Table S5). The majority of these unique fecalassociated OTUs were assigned to the family Neocallimastigaceae. The relative sequence abundance of shared fungal OTUs were not different between the two efficiency groups (White's nonparametric $t$-test, $P>0.05$, Supplementary Table S6).

\section{DISCUSSION}

The GIT of animals is differentiated anatomically into defined and adapted regions (stomach, SI and large intestine) that are colonized by microorganisms capable of metabolizing ingested dietary substrates (Turnbaugh and Gordon, 2009). Ruminants have forestomachs (rumen, reticulum, and omasum) that are responsible for the digestion and conversion of most dietary components into VFAs that represent the major source of energy for ruminants (Russell and Hespell, 1981; Jami and Mizrahi, 2012). Because the rumen is the primary site of feed fermentation, most studies evaluating the ruminant microbiome have focused on the ruminal ecosystem (Tajima et al., 2001; Pitta et al., 2010; Li et al., 2012; Firkins and Yu, 2015; Shabat et al., 2016; Li and Guan, 2017). However, studies have demonstrated relevant associations between the GIT microbiota and ruminant production (Myer et al., 2015b,c, 2016, Lindholm-Perry et al., 2016). Although it has been shown that different portions of the GIT harbor distinct microbial communities with different biochemical properties, relevant species of microorganisms are 


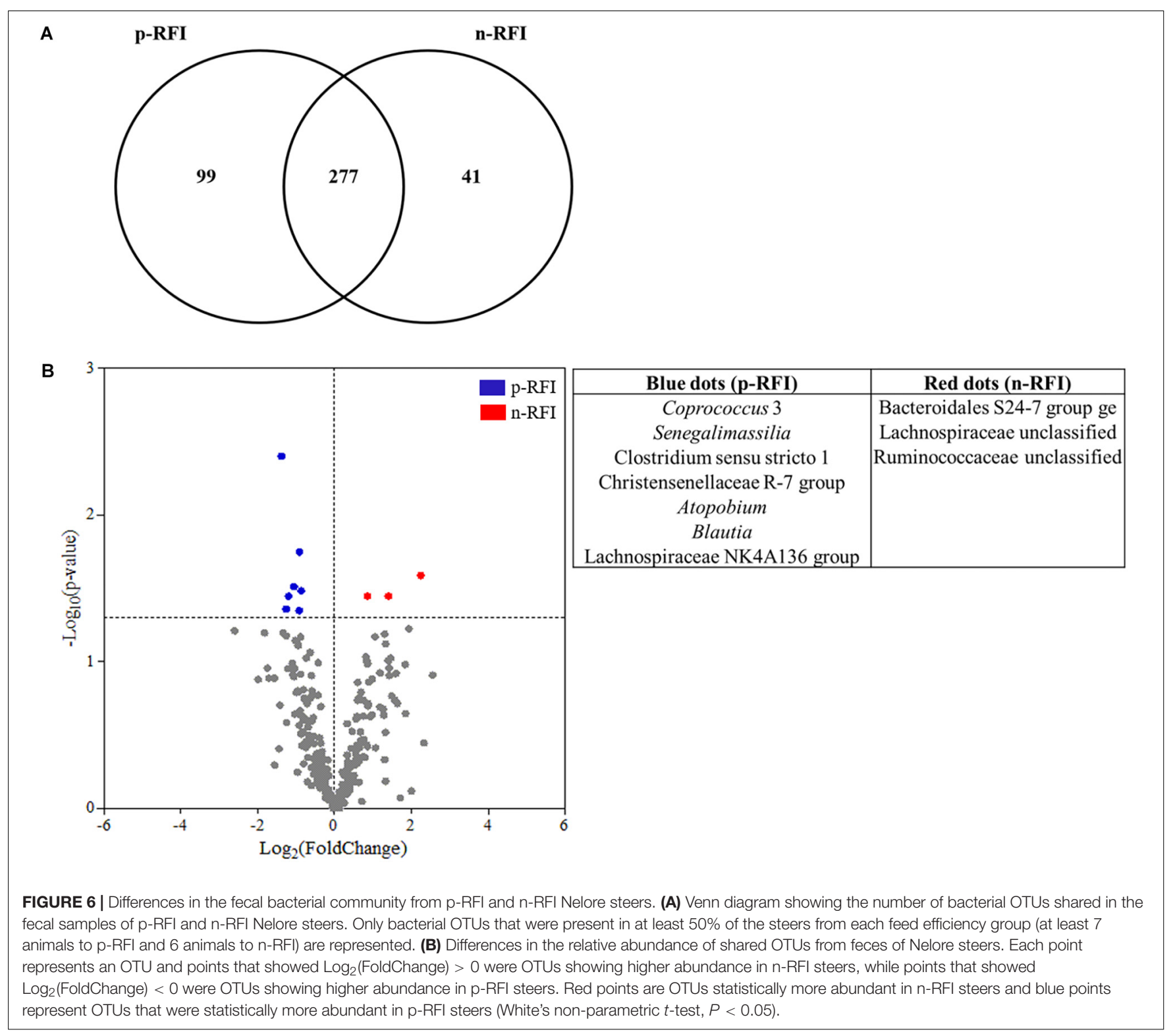

known to be shared across all GIT regions (Frey et al., 2009; Oliveira et al., 2013).

In this study, we characterized the bacterial and fungal composition of the GIT of 27 Nelore steers, an important beef cattle breed due to its prominence in world meat production. Our data revealed significant differences in the bacterial and fungal community (beta diversity) between the evaluated segments (Figure 2) and supports the idea that passage of digesta through adjacent GIT sections affects the composition of the gut microbial communities (Oliveira et al., 2013; Mao et al., 2015; Perea et al., 2017). Mao et al. (2015) characterized the bacterial communities colonizing different regions within the GIT of Holstein dairy cattle and reported significant differences in species composition and metabolic function of digesta-associated and mucosa-adherent microbiota. Heterogeneity in microbiota composition of distinct GIT segments have been reported not only for ruminants (Dias et al., 2017; Perea et al., 2017), but also for other animals like chickens (Clavijo and Vives Flórez, 2017) and mice (Montealegre et al., 2016).

The diversity and abundance of gut microorganisms also varies considerably according to host development and anatomical location, mainly because of varying physiochemical conditions (e.g., $\mathrm{pH}$, redox potential, oxygen availability), availability of nutrients and sites for adhesion, host secretions (mucins), and exposure to exogenous compounds that cause disturbance in the ecosystem (e.g., antibiotics, dietary changes, and pathogens) (Carbonero et al., 2014; Moya and Ferrer, 2016). In our study, several differences were observed in composition, richness (Chao index), diversity (Shannon index), and species dominance (Simpson) during the passage from the rumen to the SI. The genera Romboutsia and an unclassified Peptostreptococcaceae represented the most abundant OTUs in 


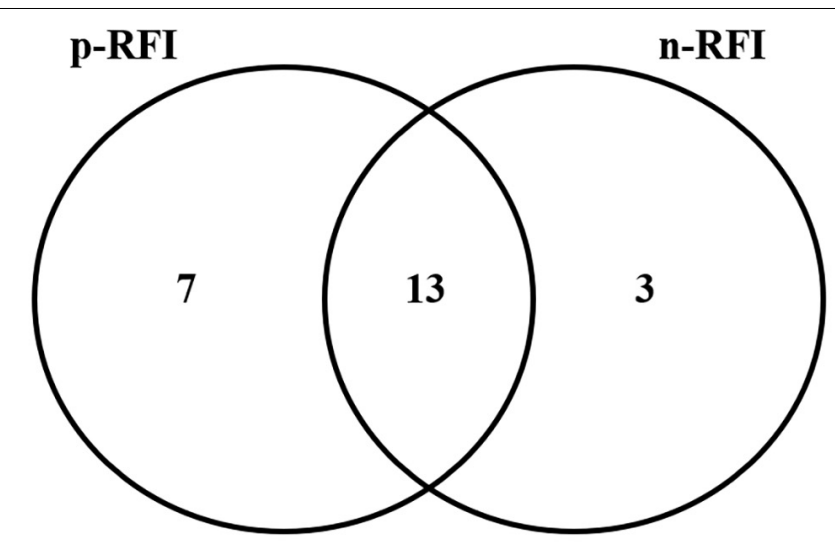

\begin{tabular}{|cc|cc|lc|}
\hline & p- RFI & Shared & \multicolumn{2}{|c|}{ n - RFI } \\
\hline Otu000043 & Fungi unclassified & Otu000001 & Orpinomyces & Otu000006 & Neocallimastigaceae \\
Otu000057 & Neocallimastix & Otu000002 & Neocallimastigaceae & Otu000027 & Fungi unclassified \\
Otu000009 & Neocallimastigaceae & Otu000003 & Orpinomyces & Otu000128 & Orpinomyces \\
Otu000077 & Fungi unclassified & Otu000004 & Neocallimastigaceae & & \\
Otu000058 & Neocallimastix & Otu000005 & Neocallimastigaceae & & \\
Otu000042 & Neocallimastigaceae & Otu000007 & Orpinomyces & & \\
Otu000113 & Fungi unclassified & Otu000008 & Anaeromyces & & \\
& & Otu000023 & Ascomycota & & \\
& Otu000031 & Orpinomyces & & \\
& Otu000044 & Anaeromyces & & \\
& Otu000056 & Fungi unclassified & & \\
& Otu000069 & Fungi unclassified & & \\
& Otu000075 & Fungi unclassified & & \\
\end{tabular}

FIGURE 7 | Venn diagram showing the number of fungal OTUs shared in the feces of p-RFI and n-RFI Nelore steers. Only fungal OTUs that were present in at least $50 \%$ of the steers from each feed efficiency group (7 animals in p-RFI and 6 animals in n-RFI) are represented.

the SI, but they were not present in other regions of the Nelore GIT. Members of the Lachnospiraceae NK3A20 group (OTUs OTU00009 and OTU000020) were also abundant in the SI and were also present in the rumen, cecum and fecal samples.

These differences in the microbial community across the Nelore GIT are possibly related to drastic changes in chemical (acidification and host enzymes secretion) and physical (osmolarity, adhesion sites) conditions in the abomasum (Figure 1). The secretion of enzymes by the host in the glandular stomach (abomasum) and the SI allows for digestion of microbial biomass, which provides protein to the host (Russell and Hespell, 1981). In addition, feed digestion in the SI is shorter than in the rumen due to a faster passage rate, which limits the establishment and adaptation of the microbiota to the physical and chemical conditions present in this portion of the GIT (Frey et al., 2009; Carbonero et al., 2014). This is reflected in the SI, which had the lowest total VFA concentration of all GIT segments analyzed in this study (Table 1). Microbial fermentation appears to be reestablished in the large intestine, with the proportion of acetate, propionate and butyrate being similar to the rumen (Table 2), which agrees with our observations that microbial diversity increases in the distal segments of the GIT (Figure 1).

Despite the divergences observed among the microbial communities of the GIT segments, the bacterial families
Lachnospiraceae and Ruminococcaceae (Figure 3) and the fungal family Neocallimastigaceae (Table 1) were the most abundant across the GIT. The Lachnospiraceae includes species of bacteria with fibrolytic and proteolytic properties, such as Lachnospira multiparus and Butyrivibrio fibrisolvens, while members of the Ruminococcaceae family include cellulolytic bacteria, such as Ruminococcus albus and R. flavefaciens (Russell and Rychlik, 2001). The Neocallimastigaceae family comprises several anaerobic fungal species (e.g., Orpinomyces, Anaeromyces, Neocallimastix, and Piromyces) and recent transcriptomic and comparative genomics studies indicated that these species produce highly active enzymes for the degradation of plant cell walls and recalcitrant fiber in the rumen (Haitjema et al., 2017; Gruninger et al., 2018). Considering the functional role of these microbial groups in the degradation of plant biomass, their relative abundance in the GIT segments may represents mechanisms for additional energy acquisition by the host through the digestion of structural carbohydrates that escape ruminal degradation (Oliveira et al., 2013; Myer et al., 2015c). Moreover, our finding that 28 bacterial and 6 fungal OTUs were common to all segments of the GIT (Figure 5) suggest that some bacterial and fungal species are able to colonize and/or survive in all portions of the GIT. All shared bacterial OTUs represented Gram-positives and were found in greater abundances in the 
SI. The digestive conditions and processes that occur in the transition from the abomasum to the SI could function as a filter that limits the growth of ruminal microorganisms throughout the entire ruminant GIT (Oliveira et al., 2013; Moya and Ferrer, 2016; Myer et al., 2016). In the absence of the enzymatic and chemical processes that occur in the abomasum and the SI, the composition of microbial communities would probably be much less divergent across the GIT.

Sampling GIT contents often relies upon invasive techniques or sacrificing the animal, and some studies have suggested the use of fecal samples as a proxy to explore potential associations between the GIT microbiota and host phenotype (Turnbaugh and Gordon, 2009; Tajima et al., 2012; Nash et al., 2017; Tang et al., 2018). Here, we evaluated the relationship between fecal microbiota composition and feed efficiency and found that differences between bacterial communities of negative (n-RFI) and positive (p-RFI) feed efficiencient steers are due to OTUs belonging to the families Lachnospiraceae (Blautia, Coprococcus, Butyrivibrio, and Roseburia genera), Prevotelaceae (Alloprevotella and Prevotella genera), Coriobacteriaceae (Atopodium and Senegalimassilia genera), and Ruminococcaceae (Saccharofermentans genus) (Supplementary Tables S3, S4). These bacteria are typically involved in the degradation of dietary substrates and VFA production in ruminants (Russell and Rychlik, 2001; Jami and Mizrahi, 2012; Li and Guan, 2017). For example, species within the Prevotella and Butyrivibrio are well known for producing hydrolytic enzymes, such as cellulases, xylanases, and beta-glucanases, that degrade plant structural polysaccharides (Cotta and Hespell, 1986; Kamra, 2005). Furthermore, Butyrivibrio is involved in butyrate production, which represents one of the main sources of energy for enterocytes and exerts strong effects on GIT epithelial cells, such as stimulation of cell proliferation and differentiation (Wächtershäuser and Stein, 2000; Guilloteau et al., 2010). Butyrate also has an effect on the expression of leptin in bovine adipocytes, affecting feed intake and energy expenditure of the host (Soliman et al., 2007). In addition, ruminal abundance of members of the Lachnospiraceae and Ruminococcaceae families, as well as the genus Prevotella, have been reported as being associated with host feed efficiency (Carberry et al., 2012; McCann et al., 2014; Myer et al., 2015a; Shabat et al., 2016). For the fungal communities, shared OTUs did not vary in abundance between the p-RFI and n-RFI groups (Supplementary Table S6), with only a few unique OTUs identified for each group. Studies on anaerobic fungi are challenging due to their fastidious nature and information about their potential role in feed efficiency is lacking (Haitjema et al., 2017; Gruninger et al., 2018).

The results presented here expand our knowledge about the composition of the Nelore GIT microbiome and suggests specific taxa potentially associated with feed efficiency. However, it should be noted that these findings are observational, precluding inferences on causality. We observed differences between microbial communities colonizing adjacent sections of the Nelore GIT and showed that fecal samples, although distinct in composition from other GIT sections, harbor considerable microbial diversity associated with different fermentation products and feed efficiency phenotypes. This is promising, as collection of fecal samples is non-invasive and more practical for sampling large numbers of animals to study different host traits. For example, analysis of fecal samples has been applied to population-scale studies of humans, making it possible to associate changes in the GIT microbiota with the health status of individuals and several physiological and psychological conditions, including obesity, autism, humor, kidney stone, biogeography, and eating habits (Turnbaugh and Gordon, 2009; Lloyd-Price et al., 2017; Nash et al., 2017; Tang et al., 2018).

Thus, while additional studies are needed, these findings suggest that analysis of microbial communities in fecal samples could be useful for monitoring functional groups related to feed efficiency in cattle. Further confirmatory studies using larger cohorts will be needed to corroborate our findings and to evaluate the consistency in the relationship of fecal microbial populations with animal phenotype. Large-scale studies assessing the fecal microbial community of cattle herds could enable the identification of microbial groups potentially associated with a causal effect on ruminant feed efficiency. Expanding knowledge of the effects of the microbial community on the well-being and health of cattle will also be important for the development of new management, nutrition and manipulation strategies of the GIT microbial community.

\section{ETHICS STATEMENT}

The experimental procedures were approved by the Ethics Committee on Animal Use of the Instituto de Zootecnia (CEUAIZ, Protocol 213-15), in accordance with guidelines of São Paulo State Law No. 11.977, Brazil, and by the Ethics Committee on Production Animal Use of the Universidade Federal de Viçosa (CEUAP-UFV, Protocol 026/2015).

\section{AUTHOR CONTRIBUTIONS}

DL, GS, ED, and HM, conceived and designed the experiments. GS, MM, SB, ED, MSD, and HM provided experimental and laboratorial resources. DL, MSD, and HM conducted sample collection and DNA extraction. DL, CB, and AL performed library construction and sequencing. DL, AL, GS, and HM conducted data analyses and interpretation of results. DL and HM wrote the manuscript. AL, GS, MSD, and ED reviewed and edited the manuscript. All authors read and approved the final manuscript.

\section{FUNDING}

This study was supported by FAPEMIG (APQ-02171-15), CNPq (PVE 313792/2014-3), CAPES (Proex PPGMBA/UFV), and the INCT Ciência Animal. This work was also supported by a traineeship from the National Institute of Allergy and Infectious Diseases of the National Institutes of Health under Award Number T32AI55397 to AL. 


\section{ACKNOWLEDGMENTS}

We would like to thank the IZ staff for their support and help in handling the animals and Joseph Skarlupka (Suen laboratory) for his support in laboratory and sequencing analysis.

\section{SUPPLEMENTARY MATERIAL}

The Supplementary Material for this article can be found online at: https://www.frontiersin.org/articles/10.3389/fmicb. 2019.01263/full\#supplementary-material

FIGURE S1 | Non-metric multidimensional scaling (nMDS) plots of the Bray-Curtis dissimilarity index for bacterial (A) and fungal (B) communities in fecal samples of Nelore steers. Individual points represent fecal samples from different steers, with

\section{REFERENCES}

Associação Brasileira das Indústrias Exportadoras de Carnes [ABIEC] (2017) Available at: http://www.abiec.com.br/download/Anual-jan-dez-2017.pdf (accessed May 29, 2019).

Benatti, J. M. B., Alves Neto, J. A., de Oliveira, I. M., de Resende, F. D., and Siqueira, G. R. (2017). Effect of increasing monensin sodium levels in diets with virginiamycin on the finishing of Nellore cattle. Anim. Sci. J. 88, 1709-1714. doi: $10.1111 /$ asj.12831

Brasil (2017). Regulamento da inspeção industrial e sanitária de produtos de origem animal. Ministério da Agricultura, Pecuária e Abastecimento. Available at: https://www.saude.rj.gov.br/comum/code/MostrarArquivo.php? $\mathrm{C}=\mathrm{NzU} 2 \mathrm{NQ} \% 2 \mathrm{C} \% 2 \mathrm{C} \& \mathrm{C}=\mathrm{NzU} 2 \mathrm{NQ} \% 2 \mathrm{C} \% 2 \mathrm{C}$ (accessed February 5, 2018).

Carberry, C. A., Kenny, D. A., Han, S., McCabe, M. S., and Waters, S. M. (2012). Effect of phenotypic residual feed intake and dietary forage content on the rumen microbial community of beef cattle. Appl. Environ. Microbiol. 78, 4949-4958. doi: 10.1128/AEM.077597711

Carbonero, F., Oakley, B. B., and Purdy, K. J. (2014). Metabolic flexibility as a major predictor of spatial distribution in microbial communities. PLoS One 9:e85105. doi: 10.1371/journal.pone.0085105

Carvalho, V. V., Paulino, M. F., Detmann, E., Chizzotti, M. L., Martins, L. S., Silva, A. G., et al. (2017). Effects of supplements containing different additives on nutritional and productive performance of beef cattle grazing tropical grass. Trop. Anim. Health Prod. 49, 983-988. doi: 10.1007/s11250-017-12861288

Cho, I., and Blaser, M. J. (2012). applications of next-generation sequencing the human microbiome: at the interface of health and disease. Nat. Rev. Genet. 13 260-270. doi: $10.1038 / \mathrm{nrg} 3182$

Clavijo, V., and Vives Flórez, M. J. (2017). The gastrointestinal microbiome and its association with the control of pathogens in broiler chicken production: a review. Poult. Sci. 97, 1006-1021. doi: 10.3382/ps/pe x359

Cotta, M., and Hespell, R. (1986). Proteolytic activity of the ruminal bacterium butyrivibrio fibrisolvens. Appl. Environ. Microbiol. Microbiol. 52, $51-58$.

Crossland, W. L., Tedeschi, L. O., Callaway, T. R., Miller, M. D., Smith, W. B., and Cravey, M. (2017). Effects of rotating antibiotic and ionophore feed additives on volatile fatty acid production, potential for methane production, and microbial populations of steers consuming a moderate-forage diet. J. Anim. Sci. 95, 4554-4567. doi: 10.2527/jas2017.1665

Dias, J., Marcondes, M. I., Noronha, M. F., Resende, R. T., Machado, F. S., Mantovani, H. C., et al. (2017). Effect of pre-weaning diet on the ruminal archaeal, bacterial, and fungal communities of dairy calves. Front. Microbiol. 8:1553. doi: 10.3389/fmicb.2017. 01553 the green and purple dots representing the $n-R F I$ and the $p-R F$ steers, respectively.

TABLE S1 | Summary of sequencing data derived from GIT contents of Nelore steers.

TABLE S2 | Alpha-diversity metrics of the bacterial community in fecal samples of Nelore steers according to RFI group.

TABLE S3 | Most abundant exclusive bacterial OTUs identified in fecal samples of Nelore steers showing low ( $p-R F I)$ or high ( $n-R F I)$ feed efficiency.

TABLE S4 | Shared bacterial OTUs that were more abundant (White's non-parametric $t$-test, $P<0.05$ ) in fecal samples of Nelore steers showing n-RFI (gray lines) or $\mathrm{p}-\mathrm{RFI}$ (white lines).

TABLE S5 | Fungal OTUs that were unique to the fecal-associated microbiome of Nelore steers showing low ( $p-R F I)$ or high ( $n-R F I)$ feed efficiency.

TABLE S6 | Shared fungal OTUs from the fecal microbiome of Nelore steers showing $n-R F I$ and $p-R F I$.

Edgar, R. C., Haas, B. J., Clemente, J. C., Quince, C., and Knight, R. (2011). UCHIME improves sensitivity and speed of chimera detection. Bioinformatics 27, 2194-2200. doi: 10.1093/bioinformatics/btr381

Food and Agriculture Organization of the United Nations [FAO] (2016). Available at: http://www.fao.org/ag/againfo/themes/en/meat/home.html (Accessed October 10, 2018).

Fidelis, H. A., Bonilha, S. F. M., Tedeschi, L. O., Branco, R. H., Cyrillo, J. N. S. G., and Mercadante, M. E. Z. (2017). Residual feed intake, carcass traits and meat quality in Nellore cattle. Meat. Sci. 128, 34-39. doi: 10.1016/j.meatsci.2017. 02.004

Firkins, J. L., and Yu, Z. (2015). Ruminant nutrition symposium: how to use data on the rumen microbiome to improve our understanding of ruminant nutrition. J. Anim. Sci. 93, 1450-1470. doi: 10.2527/jas.20148754

Frey, J. C., Pell, A. N., Berthiaume, R., Lapierre, H., Lee, S., Ha, J. K., et al. (2009). Comparative studies of microbial populations in the rumen, duodenum, ileum and faeces of lactating dairy cows. J. Appl. Microbiol. 108, 1982-1993. doi: 10.1111/j.1365-2672.2009. $04602 \mathrm{x}$

Gruninger, R. J., Nguyen, T. T. M., Reid, I. D., Yanke, J. L., Wang, P., Abbott, D. W., et al. (2018). Application of transcriptomics to compare the carbohydrate active enzymes that are expressed by diverse genera of anaerobic fungi to degrade plant cell wall carbohydrates. Front. Microbiol. 9:1581. doi: 10.3389/fmicb.2018. 01581

Guilloteau, P., Martin, L., Eeckhaut, V., Ducatelle, R., Zabielski, R., and Van Immerseel, F. (2010). From the gut to the peripheral tissues: the multiple effects of butyrate. Nutr. Res. Rev. 23, 366-384. doi: 10.1017/S0954422410 000247

Haitjema, C. H., Gilmore, S. P., Henske, J. K., Solomon, K. V., de Groot, R., Kuo, A., et al. (2017). A parts list for fungal cellulosomes revealed by comparative genomics. Nat. Microbiol. 2:17087. doi: 10.1038/nmicrobiol.2017.87

Hammer, O., Harper, D. A. T., and Ryan, P. D. (2001). PAST: paleontological statistics software package for education and data analysis. Palaeont. electron. 4, 1-9.

Henderson, G., Cox, F., Kittelmann, S., Miri, V. H., Zethof, M., Noel, S. J., et al. (2013). Effect of DNA extraction methods and sampling techniques on the apparent structure of cow and sheep rumen microbial communities. PLoS One 8:e74787. doi: 10.1371/journal.pone.0074787

Jami, E., and Mizrahi, I. (2012). Composition and similarity of bovine rumen microbiota across individual animals. PLoS One 7:e33306. doi: 10.1371/journal. pone.0033306

Jewell, K. A., McCormick, C. A., Odt, C. L., Weimer, P. J., and Suen, G. (2015). Ruminal bacterial community composition in dairy cows is dynamic over the course of two lactations and correlates with feed efficiency. Appl. Environ. Microbiol. 81, 4697-4710. doi: 10.1128/AEM.007 $20-715$ 
Jose Neto, A., Zervoudakis, J. T., da Silva-Marques, R. P., Silva, L. C. R. P., Hatamoto-Zervoudakis, L. K., and Klopfenstein, T. J. (2016). Suitable strategy to improve nitrogen utilization and reduce the environmental impact of Nellore bulls supplemented on tropical pasture. J. Anim. Sci. 94, 1110-1122. doi: 10. 2527/jas.2015-2030

Kamra, D. N. (2005). Rumen microbial ecosystem. Curr. Sci. 89, 124-135. doi: 10.1146/annurev.es.06.110175.000351

Kittelmann, S., Seedorf, H., Walters, W. A., Clemente, J. C., Knight, R., Gordon, J. I., et al. (2013). Simultaneous amplicon sequencing to explore cooccurrence patterns of bacterial, archaeal and eukaryotic microorganisms in rumen microbial communities. PLoS One 8:e47879. doi: 10.1371/journal.pone. 0047879

Kõljalg, U., Nilsson, R. H., Abarenkov, K., Tedersoo, L., Taylor, A. F. S., Bahram, M., et al. (2013). Towards a unified paradigm for sequencebased identification of fungi. Mol. Ecol. 22, 5271-5277. doi: 10.1111/mec. 12481

Kozich, J. J., Westcott, S. L., Baxter, N. T., Highlander, S. K., and Schloss, P. D. (2013). Development of a dual-index sequencing strategy and curation pipeline for analyzing amplicon sequence data on the MiSeq illumina sequencing platform. Appl. Environ. Microbiol. 79, 5112-5120. doi: 10.1128/AEM.010431013

Krause, D. O., and Russell, J. B. (1996). How many ruminal bacteria are there? J. Dairy Sci. 79, 1467-1475. doi: 10.3168/jds.S0022-0302(96)7650676502

Ley, R. E., Turnbaugh, P. J., Klein, S., and Gordon, J. I. (2006). Microbial ecology: human gut microbes associated with obesity. Nature 444, 1022-1023. doi: 10. 1038/4441022a

Li, F., and Guan, L. L. (2017). Metatranscriptomic profiling reveals linkages between the active rumen microbiome and feed efficiency in beef cattle. Appl. Environ. Microbiol. 83:e61-17. doi: 10.1128/AEM.000 61-17

Li, R. W., Connor, E. E., Li, C., Baldwin Vi, R. L., and Sparks, M. E. (2012). Characterization of the rumen microbiota of pre-ruminant calves using metagenomic tools. Environ. Microbiol. 14, 129-139. doi: 10.1111/j.1462-2920. 2011.02543.x

Lindholm-Perry, A. K., Butler, A. R., Kern, R. J., Hill, R., Kuehn, L. A., Wells, J. E., et al. (2016). Differential gene expression in the duodenum, jejunum and ileum among crossbred beef steers with divergent gain and feed intake phenotypes. Anim. Genet. 47, 408-427. doi: 10.1111/age.12440

Lloyd-Price, J., Mahurkar, A., Rahnavard, G., Crabtree, J., Orvis, J., Hall, A. B., et al. (2017). Strains, functions and dynamics in the expanded human microbiome project. Nature 550, 61-66. doi: 10.1038/nature23889

Mao, S., Zhang, M., Liu, J., and Zhu, W. (2015). Characterising the bacterial microbiota across the gastrointestinal tracts of dairy cattle: membership and potential function. Sci. Rep. 5:16116. doi: 10.1038/srep 16116

McCann, J. C., Wiley, L. M., Forbes, T. D., Rouquette, F. M., and Tedeschi, L. O. (2014). Relationship between the rumen microbiome and residual feed intake-efficiency of brahman bulls stocked on bermudagrass pastures. PLoS One 9:e91864. doi: 10.1371/journal.pone.0091864

Montealegre, M. C., Singh, K. V., and Murray, B. E. (2016). Gastrointestinal tract colonization dynamics by different enterococcus faecium clades. J. Infect. Dis. 213, 1914-1922. doi: 10.1093/infdis/jiv597

Moya, A., and Ferrer, M. (2016). Functional redundancy-induced stability of gut microbiota subjected to disturbance. Trends Microbiol. 24, 402-413. doi: 10. 1016/j.tim.2016.02.002

Myer, P. R., Smith, T. P. L., Wells, J. E., Kuehn, L. A., and Freetly, H. C. (2015a). Rumen microbiome from steers differing in feed efficiency. PLoS One 10:e0129174. doi: 10.1371/journal.pone.0129174

Myer, P. R., Wells, J. E., Smith, T. P. L., Kuehn, L. A., and Freetly, H. C. (2015b). Cecum microbial communities from steers differing in feed efficiency. J. Anim. Sci. 93, 5327-5340. doi: 10.2527/jas2015-9415

Myer, P. R., Wells, J. E., Smith, T. P. L., Kuehn, L. A., and Freetly, H. C. (2015c). Microbial community profiles of the colon from steers differing in feed efficiency. Springerplus 4:454. doi: 10.1186/s40064-015-12011206

Myer, P. R., Wells, J. E., Smith, T. P. L., Kuehn, L. A., and Freetly, H. C. (2016). Microbial community profiles of the jejunum from steers differing in feed efficiency. J. Anim. Sci. 94, 327-338. doi: 10.2527/jas.20159839

Nash, A. K., Auchtung, T. A., Wong, M. C., Smith, D. P., Gesell, J. R., Ross, M. C., et al. (2017). The gut mycobiome of the human microbiome project healthy cohort. Microbiome 5:153. doi: 10.1186/s40168-017-03 73-374

Nkrumah, J. D., Okine, E. K., Mathison, G. W., Schmid, K., Li, C., Basarab, J. A., et al. (2006). Relationships of feedlot feed efficiency, performance, and feeding behavior with metabolic rate, methane production, and energy partitioning in beef cattle1. J. Anim. Sci. 84, 145-153. doi: 10.2527/2006.84 $1145 \mathrm{x}$

Oliveira, M. N. V., Jewell, K. A., Freitas, F. S., Benjamin, L. A., Tótola, M. R., Borges, A. C., et al. (2013). Characterizing the microbiota across the gastrointestinal tract of a Brazilian Nelore steer. Vet. Microbiol. 164, 307-314. doi: 10.1016/j. vetmic.2013.02.013

Parks, D. H., Tyson, G. W., Hugenholtz, P., and Beiko, R. G. (2014). STAMP: statistical analysis of taxonomic and functional profiles. Bioinformatics 30, 3123-3124. doi: 10.1093/bioinformatics/btu494

Perea, K., Perz, K., Olivo, S. K., Williams, A., Lachman, M., Ishaq, S. L., et al. (2017). Feed efficiency phenotypes in lambs involve changes in ruminal, colonic, and small-intestine-located microbiota. J. Anim. Sci. 95, 2585-2592. doi: 10.2527/ jas2016.1222

Pitta, D. W., Pinchak, W. E., Dowd, S. E., Osterstock, J., Gontcharova, V., Youn, E., et al. (2010). Rumen bacterial diversity dynamics associated with changing from bermudagrass hay to grazed winter wheat diets. Microb. Ecol. 59, 511-522. doi: 10.1007/s00248-009-9609-9606

Quast, C., Pruesse, E., Yilmaz, P., Gerken, J., Schweer, T., Yarza, P., et al. (2012). The SILVA ribosomal RNA gene database project: improved data processing and web-based tools. Nucleic Acids Res. 41, D590-D596. doi: 10.1093/nar/gks 1219

Russell, J. B., and Hespell, R. B. (1981). Microbial rumen fermentation. J. Dairy Sci. 64, 1153-1169. doi: 10.3168/jds.S0022-0302(81)82 694-X

Russell, J. B., and Rychlik, J. L. (2001). Factors that alter rumen microbial ecology. Science 292, 1119-1122. doi: 10.1126/science. 1058830

Sasson, G., Ben-Shabat, S. K., Seroussi, E., Doron-Faigenboim, A., Shterzer, N., Yaacoby, S., et al. (2017). Heritable bovine rumen bacteria are phylogenetically related and correlated with the Cow's capacity to harvest. mBio 8, e703-e717. doi: 10.1128/mBio.00703-17

Schloss, P. D., Westcott, S. L., Ryabin, T., Hall, J. R., Hartmann, M., Hollister, E. B., et al. (2009). Introducing mothur: open-source, platform-independent, community-supported software for describing and comparing microbial communities. Appl. Environ. Microbiol. 75, 7537-7541. doi: 10.1128/AEM. 01541-09

Shabat, S. K., Sasson, G., Doron-Faigenboim, A., Durman, T., Yaacoby, S., Berg Miller, M. E., et al. (2016). Specific microbiome-dependent mechanisms underlie the energy harvest efficiency of ruminants. ISME J. 10, 2958-2972. doi: 10.1038/ismej.2016.62

Siegfried, R., Ruckemann, H., Stumpf, G., Siegfried, V. R., Ruckermann, H., and Siegfried, B. D. (1984). Method for the determination of organic acids in silage by high performance liquid chromatography. Landwirt Forsch 37, 298-304.

Soliman, M., Kimura, K., Ahmed, M., Yamaji, D., Matsushita, Y., OkamatsuOgura, Y., et al. (2007). Inverse regulation of leptin mRNA expression by short- and long-chain fatty acids in cultured bovine adipocytes. Domest. Anim. Endocrinol. 33, 400-409. doi: 10.1016/j.domaniend.2006. 08.005

Stevenson, D. M., and Weimer, P. J. (2007). Dominance of prevotella and low abundance of classical ruminal bacterial species in the bovine rumen revealed by relative quantification real-time PCR. Appl. Microbiol. Biotechnol. 75, 165-174. doi: 10.1007/s00253-006-0802-y

Tajima, K., Aminov, R. I., and Nagamine, T. (2001). Diet-dependent shifts in the bacterial population of the rumen revealed with real-time PCR dietdependent shifts in the bacterial population of the rumen revealed with realtime PCR. Appl. Environ. Microbiol. 67, 2766-2774. doi: 10.1128/AEM.67.6. 2766

Tajima, K., Aminov, R. I., Nagamine, T., de Oliveira, M. N. V., Jewell, K. A., Freitas, F. S., et al. (2012). Commensal microbiome effects on mucosal immune 
system development in the ruminant gastrointestinal tract. J. Dairy Sci. 7, 1069-1079.

Tang, R., Jiang, Y., Tan, A., Ye, J., Xian, X., Xie, Y., et al. (2018). 16S rRNA gene sequencing reveals altered composition of gut microbiota in individuals with kidney stones. Urolithiasis 46, 503-514. doi: 10.1007/s00240-0181037-y

Turnbaugh, P. J., and Gordon, J. I. (2009). The core gut microbiome, energy balance and obesity. J. Physiol. 587, 4153-4158. doi: 10.1113/jphysiol.2009. 174136

Wächtershäuser, A., and Stein, J. (2000). Rationale for the luminal provision of butyrate in intestinal diseases. Eur. J. Nutr. 39, 164-171. doi: 10.1007/ s003940070020

Wang, Q., Garrity, G. M., Tiedje, J. M., Cole, J. R., and Al, W. E. T. (2007). Naive bayesian classifier for rapid assignment of rRNA sequences into the new bacterial taxonomy. Appl. Environ. Microbiol. 73, 5261-5267. doi: 10.1128/AEM.00062-07

Conflict of Interest Statement: The authors declare that the research was conducted in the absence of any commercial or financial relationships that could be construed as a potential conflict of interest.

Copyright (c) 2019 Lopes, La Reau, Duarte, Detmann, Bento, Mercadante, Bonilha, Suen and Mantovani. This is an open-access article distributed under the terms of the Creative Commons Attribution License (CC BY). The use, distribution or reproduction in other forums is permitted, provided the original author(s) and the copyright owner(s) are credited and that the original publication in this journal is cited, in accordance with accepted academic practice. No use, distribution or reproduction is permitted which does not comply with these terms. 